Vietnam Journal of Mechanics, VAST, Vol.39, No. 2 (2017), pp. 135-147

DOI:10.15625/0866-7136/8691

\title{
AN ISOGEOMETRIC SGBEM FOR CRACK PROBLEMS OF MAGNETO-ELECTRO-ELASTIC MATERIALS
}

\author{
Han Duc Tran ${ }^{1, *}$, Binh Huy Nguyen ${ }^{1,2}$ \\ ${ }^{1}$ Vietnamese-German University, Binh Duong, Vietnam \\ ${ }^{2}$ Institute for Continuum Mechanics, Leibniz University Hannover, Germany \\ *E-mail: han.td@vgu.edu.vn \\ Received September 15, 2016
}

\begin{abstract}
The isogeometric symmetric Galerkin boundary element method is applied for the analysis of crack problems in two-dimensional magneto-electro-elastic domains. In this method, the field variables of the governing integral equations as well as the geometry of the problems are approximated using non-uniform rational B-splines (NURBS) basis functions. The key advantage of this method is that the isogeometric analysis and boundary element method deal only with the boundary of the domain. To verify the accuracy of the proposed method, numerical examples for crack problems in infinite and finite domains are examined. It is observed that the computed generalized stress intensity factors obtained by the proposed method agree well with the exact solutions and other references.
\end{abstract}

Keywords: Isogeometric analysis, NURBS, SGBEM, crack, magneto-electro-elastic.

\section{INTRODUCTION}

Isogeometric analysis (IGA) [1] has recently received much consideration of researchers because of its capability to save significantly pre-processing time for the analysis of structures [2]. This comes from the fact that, in IGA, the basis functions employed to describe the shape of the structure are also used to approximate the field variables of the problem. Although IGA has been applied successfully for the finite element method (FEM), there still exist difficulties due to the difference between the non-volumetric geometry representation used in IGA and the volumetric discretization used in FEM. To overcome this difficulty, the combinations of IGA with other methods such as meshfree methods (e.g. [3,4]) or the extended finite element method (e.g. [5-7]) have been considered. For the same purpose, the combination of IGA and the boundary element method (BEM) seems to be a very beneficial option since BEM, in the absence of body force, just deals with the boundary of the domain. Studies of IGA-BEM can be found in [8-14]. Particularly, the combination of IGA and BEM is ideal for the analysis of crack problems due to the fact that, in BEM, there is no need to discretize the region in front of the

(c) 2017 Vietnam Academy of Science and Technology 
crack tip which is usually a challenge for FEM. A particular effective BEM formulation for the analysis of crack problems is the weakly-singular symmetric Galerkin boundary element method (SGBEM), e.g. [15-17]. In this method, the singular integrals (where the numerical evaluation of the integrals is one of challenging tasks in BEM) are regularized so that they could be evaluated accurately using a standard integration scheme. Owning these advantages, the combination of IGA and SGBEM should be a powerful tool to analyse crack problems. However, as other BEM methods, the disadvantages of the current method are the complex integral equations that require advanced techniques to evaluate accurately, and that the dense coefficient matrix requires more time (relatively compared to FEM) to solve the system of discretized equations.

For magneto-electro-elastic materials, due to the complicated essence of the governing equations of field quantities, analytical solutions for crack problems are just limited to very simple geometries and loadings, e.g. [18-21]. Therefore, computational techniques are very necessary and practical for crack problems of complicated geometries, e.g. [22-26]. This paper is the extension of the IGA-SGBEM which is developed in [27] for isotropic materials to magneto-electro-elastic materials.

\section{FORMULATION}

This section presents an introduction on the equations that govern a crack problem in two-dimensional domains of magneto-electro-elastic materials. We start with the equilibrium and constitutive equations for a general boundary value problem. The governing equations for a crack problem are then introduced.

\subsection{Governing equations in magneto-electro-elastic media}

With respect to a Cartesian coordinate system where $\left\{x_{1}, x_{2}\right\}$ are the in-plane coordinates and $\left\{x_{3}\right\}$ is the out-of-plane coordinate, the setting for this work is two-dimensional in the sense that all field quantities are assumed to be independent of $x_{3}$, and as such we describe the domain occupied by the body simply in terms of a region $\Omega$ belong to the $x_{1}-x_{2}$ plane. For every point $x \in \Omega$, and with the assumption of no body force, electric charge and electric current, the field quantities are taken to be governed by equilibrium equations ${ }^{1}$

$$
\begin{aligned}
& \frac{\partial}{\partial x_{\alpha}} \sigma_{\alpha j}(x)=0 \\
& \frac{\partial}{\partial x_{\alpha}} D_{\alpha}(x)=0 \\
& \frac{\partial}{\partial x_{\alpha}} B_{\alpha}(x)=0
\end{aligned}
$$

\footnotetext{
${ }^{1}$ Here and what follows, Greek indices range from 1 to 2 , lower case Latin indices range from 1 to 3 ; repeated indices imply summation over their range.
} 
and constitutive relations

$$
\begin{aligned}
\sigma_{\alpha j}(\boldsymbol{x}) & =C_{\alpha j k \beta} \frac{\partial}{\partial x_{\beta}} u_{k}(\boldsymbol{x})+e_{\beta \alpha j} \frac{\partial}{\partial x_{\beta}} \phi(x)+h_{\beta \alpha j} \frac{\partial}{\partial x_{\beta}} \psi(\boldsymbol{x}) \\
D_{\alpha}(\boldsymbol{x}) & =e_{\alpha k \beta} \frac{\partial}{\partial x_{\beta}} u_{k}(\boldsymbol{x})-\kappa_{\alpha \beta} \frac{\partial}{\partial x_{\beta}} \phi(\boldsymbol{x})-\beta_{\alpha \beta} \frac{\partial}{\partial x_{\beta}} \psi(\boldsymbol{x}) \\
B_{\alpha}(\boldsymbol{x}) & =h_{\alpha k \beta} \frac{\partial}{\partial x_{\beta}} u_{k}(\boldsymbol{x})-\beta_{\alpha \beta} \frac{\partial}{\partial x_{\beta}} \phi(\boldsymbol{x})-\gamma_{\alpha \beta} \frac{\partial}{\partial x_{\beta}} \psi(\boldsymbol{x}) .
\end{aligned}
$$

In Eqs. (1) and (2), the notations used for the field variables are as follows. The mechanical stress and displacement are $\sigma_{\alpha j}$ and $u_{k}$ respectively, the electric induction and potential are $D_{\alpha}$ and $\phi$ respectively, and the magnetic induction and potential are $B_{\alpha}$ and $\psi$ respectively. The material is characterized by the elastic modulus $C_{\alpha j k \beta}$, piezoelectric and piezomagnetic constants $e_{\beta \alpha j}$ and $h_{\beta \alpha j}$ respectively, dielectric constant $\kappa_{\alpha \beta}$, electro magnetic constant $\beta_{\alpha \beta}$ and magnetic permeability $\gamma_{\alpha \beta}$. It is well-known that Eqs. (1) and (2) can be written in contracted forms in terms of 'generalized' variables as ${ }^{2}$

$$
\begin{aligned}
\frac{\partial}{\partial x_{\alpha}} \sigma_{\alpha J}(x) & =0 \\
\sigma_{\alpha J}(x) & =E_{\alpha J K \beta} \frac{\partial}{\partial x_{\beta}} u_{K}(x) .
\end{aligned}
$$

In the above equations, the generalized stress $\sigma_{\alpha J}$, generalized displacement $u_{K}$ and generalized moduli $E_{\alpha J K \beta}$ are defined as

$$
\begin{aligned}
& \sigma_{\alpha J}= \begin{cases}\sigma_{\alpha j}, J=1,2,3 \\
D_{\alpha}, J=4 \\
B_{\alpha}, J=5\end{cases} \\
& u_{K}= \begin{cases}u_{k}, K=1,2,3 \\
\phi, & K=4 \\
\psi, & K=5\end{cases} \\
& E_{\alpha J K \beta}= \begin{cases}C_{\alpha j k \beta}, J, K=1,2,3 \\
e_{\beta \alpha j}, J=1,2,3, K=4 \\
e_{\alpha k \beta}, J=4 \\
h_{\beta \alpha j}, J=1,2,3 \\
h_{\alpha k \beta}, J=5 \\
-\kappa_{\alpha \beta}, J, K=4, K=5 \\
-\beta_{\alpha \beta}, J=4,2,3 \\
-\beta_{\beta \alpha}, J=5 \\
-\gamma_{\alpha \beta}, J, K=5 .\end{cases}
\end{aligned}
$$

\footnotetext{
${ }^{2}$ Here and what follows, upper case Latin indices range from 1 to 5 to be capable of representing generalized quantities.
} 
Based on the definition of generalized stress above, a generalized traction vector is then defined as $t_{J}=\sigma_{\alpha J} n_{\alpha}$ in which $n$ is a unit vector normal to a particular surface (represented by a planar curve owing to the two-dimensional setting) passing through a particular point $x \in \Omega$. For the present work, it is required that the generalized moduli are symmetric such that $E_{\alpha J K \beta}=E_{\beta K J \alpha}$ and that, for any nonzero vector $z \in \mathbb{R}^{2}$, the matrix $(z, z)_{J K} \equiv z_{\alpha} E_{\alpha J K \beta} z_{\beta}$ is invertible. From now on, we omit the word 'generalized' when referring to different field quantities and material constants for the sake of simplicity.

\subsection{Governing integral equations for crack problems}

We now consider the case that the domain $\Omega$ contains embedded and/or surface-breaking cracks as shown in Fig. 1. On the ordinary boundary $\Gamma_{o}=$ $\Gamma_{t} \cup \Gamma_{u}$, traction $t_{o}$ is prescribed on the portion $\Gamma_{t}$ and displacement $\boldsymbol{u}_{0}$ is prescribed on the portion $\Gamma_{u}$. The crack is described by 'upper' and 'lower' faces $\Gamma_{c}^{+}$ and $\Gamma_{c}^{-}$, respectively, which are geometrically coincident such that their unit normals (taken to direct 'outward' to the material) satisfy $\boldsymbol{n}^{+}=-\boldsymbol{n}^{-}$. On the crack faces, traction is prescribed and this traction is selfequilibrated such that $t^{+}=-t^{-}$, where $t^{+}$and $t^{-}$refer to the traction applied on $\Gamma_{c}^{+}$and $\Gamma_{c}^{-}$respectively. Due to the geometric coincidence of the crack faces, it is sufficient to represent the crack faces by just a single face, i.e. $\Gamma_{c} \equiv \Gamma_{c}^{+}$. The entire boundary of the domain is denoted as $\Gamma$, i.e. $\Gamma=\Gamma_{o} \cup \Gamma_{c}$. For this setting, a pair of weakly singular weak-form integral equations for

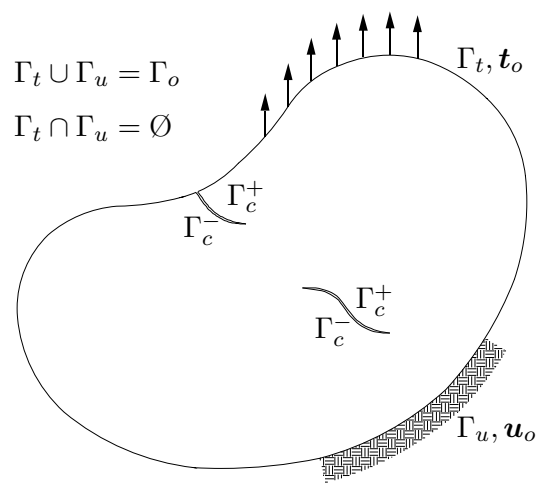

Fig. 1. Schematic of a finite domain containing embedded and surfacebreaking cracks the displacement and traction is given as [28]

$$
\begin{aligned}
\frac{1}{2} \int_{\Gamma_{o}} u_{P}(\boldsymbol{y}) \tilde{t}_{P}(\boldsymbol{y}) d s(\boldsymbol{y})= & \int_{\Gamma_{o}} \tilde{t}_{P}(\boldsymbol{y}) \int_{\Gamma_{o}} U_{J}^{P}(\boldsymbol{\xi}-\boldsymbol{y}) t_{J}(\boldsymbol{\xi}) d s(\boldsymbol{\xi}) d s(\boldsymbol{y}) \\
& +\int_{\Gamma_{o}} \tilde{t}_{P}(\boldsymbol{y}) \int_{\Gamma} G_{J}^{P}(\boldsymbol{\xi}-\boldsymbol{y}) D v_{J}(\boldsymbol{\xi}) d s(\boldsymbol{\xi}) d s(\boldsymbol{y}) \\
& -\int_{\Gamma_{o}} \tilde{t}_{P}(\boldsymbol{y}) \int_{\Gamma} n_{\alpha}(\boldsymbol{\xi}) H_{\alpha J}^{P}(\boldsymbol{\xi}-\boldsymbol{y}) v_{J}(\boldsymbol{\xi}) d s(\boldsymbol{\xi}) d s(\boldsymbol{y}), \\
-\int_{\Gamma} c(\boldsymbol{y}) t_{K}(\boldsymbol{y}) \tilde{v}_{K}(\boldsymbol{y}) d s(\boldsymbol{y})= & \int_{\Gamma} D \tilde{v}_{K}(\boldsymbol{y}) \int_{\Gamma} C_{J}^{K}(\boldsymbol{\xi}-\boldsymbol{y}) D v_{J}(\boldsymbol{\xi}) d s(\boldsymbol{\xi}) d s(\boldsymbol{y}) \\
& +\int_{\Gamma} D \tilde{v}_{K}(\boldsymbol{y}) \int_{\Gamma_{o}} G_{K}^{J}(\boldsymbol{\xi}-\boldsymbol{y}) t_{J}(\boldsymbol{\xi}) d s(\boldsymbol{\xi}) d s(\boldsymbol{y}) \\
& +\int_{\Gamma} \tilde{v}_{K}(\boldsymbol{y}) n_{\alpha}(\boldsymbol{y}) \int_{\Gamma_{o}} H_{\alpha K}^{J}(\boldsymbol{\xi}-\boldsymbol{y}) t_{J}(\boldsymbol{\xi}) d s(\boldsymbol{\xi}) d s(\boldsymbol{y}),
\end{aligned}
$$

in which $D \equiv \partial(\cdot) / \partial s$ is the differential operator with respect to the arc length, and $v_{J}$ is either the displacement or the relative crack-face displacement, $\Delta u_{J} \equiv u_{J}^{+}-u_{J}^{-}$, 
according to

$$
v_{J}(\boldsymbol{\xi})= \begin{cases}u_{J}(\boldsymbol{\xi}), & \xi \in \Gamma_{o} \\ \Delta u_{J}(\boldsymbol{\xi}), & \xi \in \Gamma_{c}\end{cases}
$$

In the above equations, $\tilde{t}_{P}$ and $\tilde{u}_{K}$ are test functions, and $\tilde{v}_{K}$ is defined as

$$
\tilde{v}_{K}(\boldsymbol{y})= \begin{cases}\tilde{u}_{K}(\boldsymbol{y}), & \boldsymbol{y} \in \Gamma_{o} \\ \Delta \tilde{u}_{K}(\boldsymbol{y}), & \mathbf{y} \in \Gamma_{c}\end{cases}
$$

The coefficient $c(\boldsymbol{y})$ is defined as $c(\boldsymbol{y})=1 / 2$ for $\boldsymbol{y} \in \Gamma_{o}$ and $c(\boldsymbol{y})=1$ for $\boldsymbol{y} \in \Gamma_{c}$. With the notation of $r$ as the distance between the source point $x$ and the field point $\xi$, i.e. $r$ is the magnitude of the vector $\boldsymbol{r} \equiv \boldsymbol{\xi}-\boldsymbol{x}$, the kernels in Eqs. (8) and (9) are defined as [28]

$$
\begin{aligned}
H_{\alpha J}^{P}(\xi-x) & =-\delta_{J P} \frac{\partial}{\partial \xi_{\alpha}}\left(\frac{\ln r}{2 \pi}\right)=-\frac{\delta_{J P}}{2 \pi} \frac{\left(\xi_{\alpha}-x_{\alpha}\right)}{r^{2}}, \\
U_{J}^{P}(\xi-x) & =K_{J \alpha}^{P \alpha}(\xi-x), \\
G_{J}^{P}(\xi-x) & =\epsilon_{\alpha \beta} E_{\alpha J K \gamma} K_{K \gamma}^{P \beta}(\xi-x), \\
C_{J}^{K}(\xi-x) & =A_{K \beta P}^{J \gamma M} K_{M \gamma}^{P \beta}(\xi-x),
\end{aligned}
$$

in which $\epsilon_{\alpha \beta}$ denotes the $2 \mathrm{D}$ alternating symbol (i.e. $\epsilon_{12}=-\epsilon_{21}=1, \epsilon_{11}=\epsilon_{22}=0$ ) and $A_{K \beta P}^{J \gamma M}$ is defined as

$$
A_{K \beta P}^{J \gamma M}=E_{v J M \gamma} E_{v K P \beta}-\frac{1}{5} E_{v J K v} E_{\beta P M \gamma}
$$

where $K_{J \beta}^{I \alpha}$ is given as

$$
K_{J \beta}^{I \alpha}(\xi-x)=-\frac{1}{4 \pi^{2}} \oint_{\|z\|=1} z_{\alpha} z_{\beta}(z, z)_{I J}^{-1} \ln |z \cdot r| d s .
$$

In Eq. (17), the contour integral is to be evaluated along a unit circle $\|z\|=1$ in the $x_{1}-x_{2}$ plane. It is noted that the kernels in Eqs. (13)-(15) are weakly singular of order $\ln r$ while the kernel in Eq. (12) is regular (see proof in [28]). The integral equations (8) and (9) are used to form a symmetric system of integral equations. Indeed, Eq. (8) is applied first on $\Gamma_{u}$ by taking $\tilde{\boldsymbol{t}}=\mathbf{0}$ on $\Gamma_{t}$. Next, Eq. (9) is applied on $\Gamma_{t}$ by taking $\tilde{\boldsymbol{v}}=\mathbf{0}$ on $\Gamma_{u} \cup \Gamma_{\mathcal{c}}$. Finally, Eq. (9) is applied on $\Gamma_{\mathcal{c}}$ by taking $\tilde{\boldsymbol{v}}=\mathbf{0}$ on $\Gamma_{u} \cup \Gamma_{t}$. The result is a system of three integral equations which are symmetric to be solved for the displacement $\boldsymbol{u}$ and the traction $\boldsymbol{t}$ on $\Gamma_{t}$ and $\Gamma_{u}$ respectively, and for the relative difference of crack-face displacement $\Delta \boldsymbol{u}$ on $\Gamma_{c}$. More details regarding the symmetry of the system of those integral equations can be found in [17].

\section{ISOGEOMETRIC SGBEM}

The system of integral equations presented in the previous section is solved approximately using the concept of isogeometric analysis [1]. Using the method of Galerkin approximation, the unknowns (i.e. the displacement $\boldsymbol{u}$, traction $\boldsymbol{t}$ and relative difference of crack-face displacement $\Delta \boldsymbol{u}$ ) are approximated using NURBS basis functions. For the 
crack, a special crack-tip element developed in [17] is employed to accurately model the asymptotic field of the crack-tip displacement. Details about the use of crack-tip element can be found in [17] and [27].

\subsection{B-Spline and NURBS}

Given a knot vector $\Xi=\left\{\zeta_{0}, \zeta_{1}, \zeta_{2}, \ldots, \zeta_{i}, \ldots, \zeta_{n+p}\right\}$ which is a set of non-decreasing real numbers $\zeta_{i}$ 's, where $n$ is the number of basis functions and $p$ is the polynomial order, the $i^{\text {th }} \mathrm{B}$-spline basis function of degree $p$ is defined recursively as

$$
\begin{aligned}
& N_{i, 0}(\zeta)= \begin{cases}1, & \text { if } \zeta_{i} \leq \zeta<\zeta_{i+1} \\
0, & \text { otherwise }\end{cases} \\
& N_{i, p}(\zeta)=\frac{\zeta-\zeta_{i}}{\zeta_{i+p}-\zeta_{i}} N_{i, p-1}(\zeta)+\frac{\zeta_{i+p+1}-\zeta}{\zeta_{i+p+1}-\zeta_{i+1}} N_{i+1, p-1}(\zeta), \text { for } p \geq 1 .
\end{aligned}
$$

With a set of B-Spline basis function $\left\{N_{i, p}\right\}_{i=1}^{n}$ and a set of control points $\{\boldsymbol{P}\}_{i=1}^{n}$ where $\boldsymbol{P} \in \mathbb{R}^{d_{s}}\left(d_{s}=2\right.$ for the present study), a B-Spline curve is then formed as

$$
\boldsymbol{C}(\zeta)=\sum_{i=1}^{n} \boldsymbol{P}_{i} N_{i, p}(\zeta)
$$

Based on the background of B-Splines, a NURBS basis function is defined as

$$
R_{i, p}(\zeta)=\frac{N_{i, p}(\zeta) w_{i}}{W(\zeta)}=\frac{N_{i, p}(\zeta) w_{i}}{\sum_{j=1}^{n} N_{j, p}(\zeta) w_{j}},
$$

where $\left\{w_{i}\right\}_{i=1}^{n}$ are the weights of the control points. Finally, a NURBS curve is defined as

$$
\boldsymbol{C}(\zeta)=\sum_{i=1}^{n} \boldsymbol{P}_{i} R_{i, p}(\zeta)
$$

where $\boldsymbol{P}_{i}$ 's are the control points.

\subsection{NURBS discretization}

The NURBS basis functions (20) are employed to approximate the trial and test functions in the governing integral equations as

$$
\begin{aligned}
& \boldsymbol{u}=\boldsymbol{\Phi}^{T} \boldsymbol{D}, \quad \tilde{\boldsymbol{u}}=\boldsymbol{\Phi}^{T} \tilde{\boldsymbol{D}} \quad \text { on } \Gamma_{t} \\
& \boldsymbol{t}=\boldsymbol{\Phi}^{T} \boldsymbol{T}, \quad \tilde{\boldsymbol{t}}=\boldsymbol{\Phi}^{T} \tilde{\boldsymbol{T}} \quad \text { on } \Gamma_{u} \\
& \Delta \boldsymbol{u}=\boldsymbol{\Phi}^{T} \Delta \boldsymbol{D}, \quad \Delta \tilde{\boldsymbol{u}}=\boldsymbol{\Phi}^{T} \Delta \tilde{\boldsymbol{D}} \quad \text { on } \Gamma_{c},
\end{aligned}
$$

where $\boldsymbol{\Phi}^{T}$ denotes the transpose of $\boldsymbol{\Phi}$ which is the column vector of local NURBS basis function (i.e. non-zero basis functions of a particular parametric element which are related to the global basis functions through a connectivity function, see [2]), $\{\boldsymbol{D}, \Delta \boldsymbol{D}, \boldsymbol{T}\}$ and $\{\tilde{D}, \Delta \tilde{D}, \tilde{T}\}$ denote the control variables of the trial and test functions, respectively. The NURBS basis functions are also used to approximate the geometry, i.e. the integration 
domain of the governing equations. For an integral of a function defined on a physical domain $\Gamma_{e} \subset \Gamma$, the integral is evaluated as

$$
\int_{\Gamma_{e}} f(x) d s=\int_{\zeta_{i}}^{\zeta_{i+1}} f(x(\zeta)) J_{\zeta} d \zeta
$$

in which the physical domain $\Gamma_{e}$ is transformed to the parametric domain $\hat{\Gamma}_{e}=\left[\zeta_{i}, \zeta_{i+1}\right]$ by the mapping (21) and the Jacobian $J_{\zeta}$ is defined as

$$
J_{\zeta}=\sqrt{\left(\frac{d x_{1}}{d \zeta}\right)^{2}+\left(\frac{d x_{2}}{d \zeta}\right)^{2}} .
$$

Since all the kernels in the governing integral equations (8) and (9) are (at most) weaklysingular kernels [28], the integral (23) is evaluated numerically using a standard scheme. Details of the numerical integration used for this study can be found in [29].

\section{NUMERICAL EXAMPLES}

This section presents the numerical examples that we analyzed in order to verify the accuracy of the developed method for crack problems in magneto-electro-elastic domains. Both infinite and finite domains are considered so that we can compare the computed results with exact solution (for the infinite-domain example) and other reference (for the finite-domain example).

\subsection{Straight crack in infinite domain}

In this example, we examine the problem of a straight crack in an infinite domain. The crack is $2 a$ long and makes an angle of $\phi$ with respect to $x_{1}$ axis as shown schematically in Fig. 2. The domain is subjected to a (mechanical) stress $\sigma_{22}=\sigma_{o}$ or an electric induction $D_{o}$ or a magnetic induction $B_{0}$ in $x_{2}$ direction at the infinity. The material employed for this example is $\mathrm{BaTiO}_{3}-\mathrm{CoFe}_{2} \mathrm{O}_{4}$ [22] with the properties shown in Tab. 1. The polling axis the material is along $x_{2}$ direction.

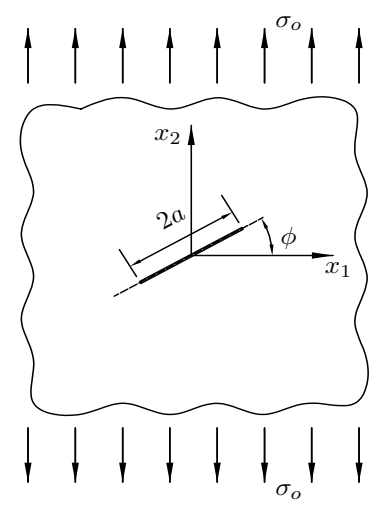

Fig. 2. Straight crack in an infinite domain
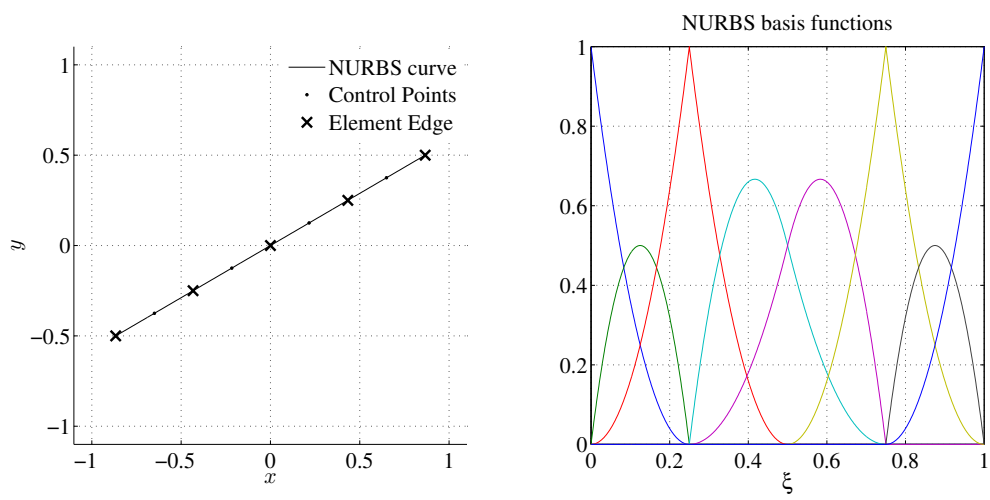

Fig. 3. NURBS discretization for the crack of the problem shown in Fig. 2 
Table 1. Material properties for $\mathrm{BaTiO}_{3}-\mathrm{CoFe}_{2} \mathrm{O}_{4}$ with $x_{2}$ being the polling direction

\begin{tabular}{|c|c|c|c|}
\hline \multicolumn{4}{|c|}{$10^{9}$} \\
\hline$C_{1111}$ & $C_{1122}$ & $C_{1133}$ & $C_{2222}$ \\
\hline 226 & 124 & 125 & 216 \\
\hline
\end{tabular}

\begin{tabular}{llllll}
\hline \multicolumn{3}{l}{ Piezoelectric constants $\left(\mathrm{C} / \mathrm{m}^{2}\right)$} & \multicolumn{3}{c}{ Piezomagnetic constants (N/Am) } \\
$e_{211}$ & $e_{222}$ & $e_{112}$ & $h_{211}$ & $h_{222}$ & $h_{112}$ \\
-2.2 & 9.3 & 5.8 & 290.2 & 350 & 275 \\
\hline
\end{tabular}

Dielectric permittivity Electromagnetic constants Magnetic permeability

$\begin{array}{llllll}\left(10^{-9} \mathrm{C} / \mathrm{Vm}\right) & \left(10^{-12} \mathrm{Ns} / \mathrm{VC}\right) & & \left(10^{-6} \mathrm{Ns}^{2} / \mathrm{C}^{2}\right) \\ \kappa_{11} & \kappa_{22} & \beta_{11} & \beta_{22} & \gamma_{11} & \gamma_{22} \\ 5.64 & 6.35 & 5.367 & 2737.5 & 297 & 83.5\end{array}$

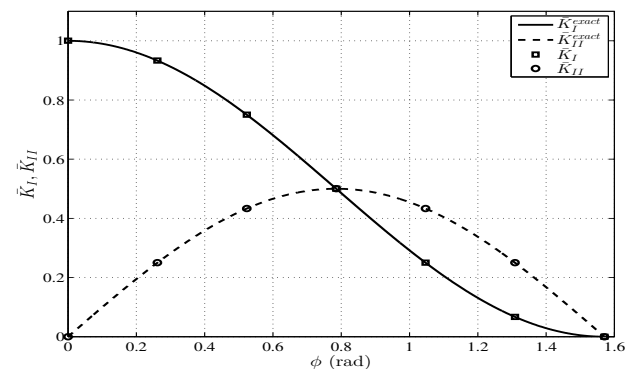

(a) Normalized stress intensity factors when $\sigma_{o}$ is applied

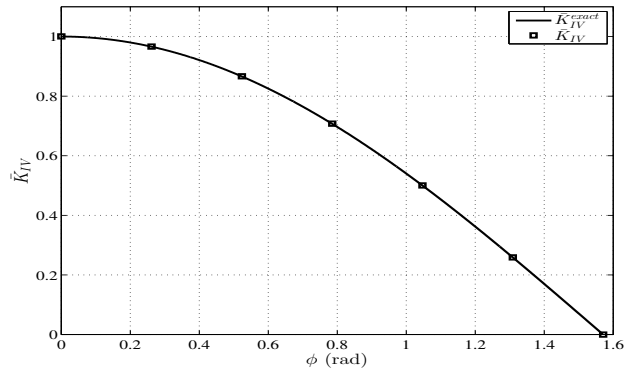

(b) Normalized electric displacement intensity factor when $D_{0}$ is applied

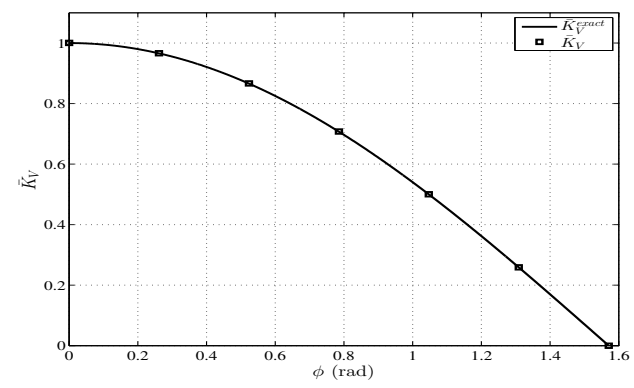

(c) Normalized magnetic induction intensity factor when $B_{0}$ is applied

Fig. 4. Normalized stress, electric displacement and magnetic induction intensity factors for the problem shown in Fig. 2 
To model the crack, we use NURBS discretization as presented in Fig. 3. For this problem, the results are uncoupled, i.e. the applied (mechanical) stress induces only stress intensity factors $K_{I}$ and $K_{I I}$, the applied electric induction induces only electric displacement intensity factor $K_{I V}$, and the applied magnetic induction induces only magnetic induction intensity factor $K_{V}$. Fig. 4 shows the computed results for $K_{I, I I}$ (normalized by $\sigma_{o} \sqrt{\pi a}$ ), $K_{I V}$ (normalized by $D_{o} \sqrt{\pi a}$ ) and $K_{V}$ (normalized by $B_{o} \sqrt{\pi a}$ ). Also in this figure, the numerical results are compared with the exact solutions given in [18]. Highly accurate numerical results are obtained.

\subsection{Edged crack in finite domain}

To verify the accuracy of the developed method for problems in a finite domain, we consider the problem of a rectangular plate with an edged crack located at the mid height of the plate as shown in Fig. 5. The plate is subjected to a uniform (mechanical) stress $\sigma_{22}=\sigma_{0}$ on the top and bottom faces. The material employed for this example is $\mathrm{BaTiO}_{3}-\mathrm{CoFe}_{2} \mathrm{O}_{4}$ [22] with the properties shown in Tab. 1. For the analysis, we employed three NURBS discretizations as shown in Fig. 6. The normalized stress intensity factor $K_{I} /\left(\sigma_{o} \sqrt{\pi a}\right)$ is shown in Tab. 2 which also presents the result obtained by [23] for the purpose of comparison. We also present the normalized electric displacement intensity factor $K_{I V} /\left(D^{*} \sqrt{\pi a}\right)$ and the magnetic induction intensity factor $K_{V} /\left(B^{*} \sqrt{\pi a}\right)$, where $D^{*}$ and $B^{*}$ are the nominal electric and magnetic induction respectively. The dimensions of $D^{*}$ and $B^{*}$ are $\mathrm{Cm}^{-2}$ and $\mathrm{N}(\mathrm{Am})^{-1}$, respectively, and their magnitudes are equal to that of $\sigma_{0}$. It is observed that our results for $K_{I}$ agree with that obtained in [23].

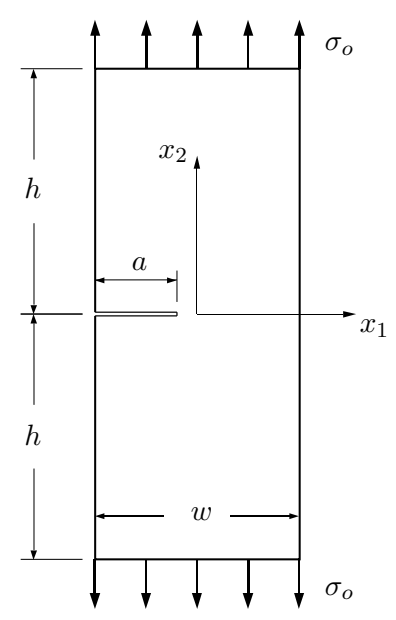

Fig. 5. Schematic of a plate with an edged crack subjected to uniform tension

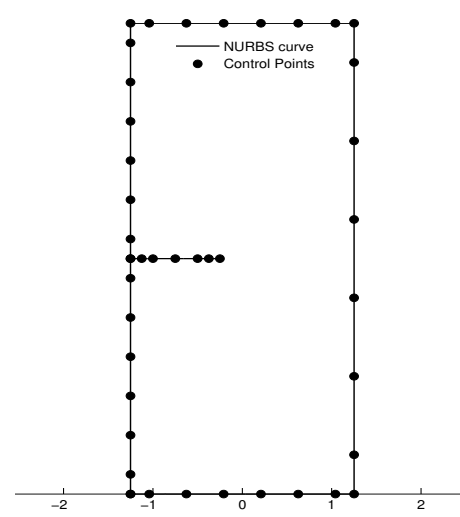

(a) Mesh 1

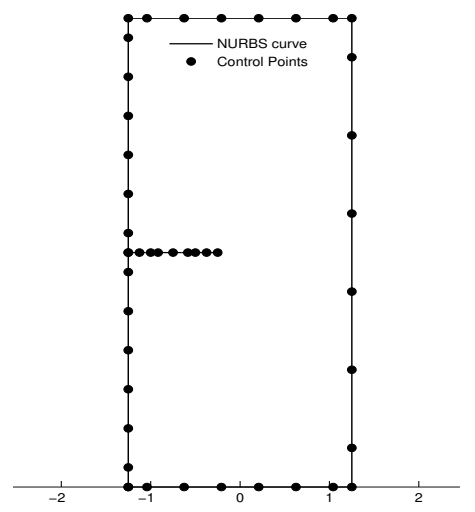

(b) Mesh 2

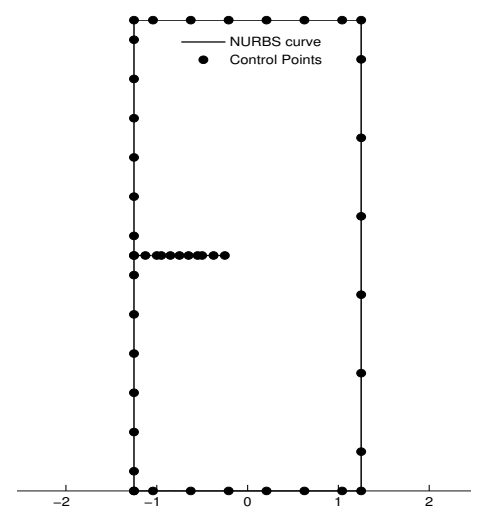

(c) Mesh 3

Fig. 6. Meshes employed for the problem shown in Fig. 5 
Table 2. Normalized stress, electric displacement and magnetic induction intensity factors for the problem shown in Fig. 5

\begin{tabular}{cccc}
\hline Mesh \# & $\frac{K_{I}}{\sigma_{0} \sqrt{\pi a}}$ & $\begin{array}{c}\frac{K_{I V}}{D^{*} \sqrt{\pi a}} \\
\left(\times 10^{-10}\right)\end{array}$ & $\begin{array}{c}\frac{K_{V}}{B^{*} \sqrt{\pi a}} \\
\left(\times 10^{-9}\right)\end{array}$ \\
\hline 1 & 2.1083 & 7.7598 & 2.1842 \\
2 & 2.1042 & 7.7444 & 2.1802 \\
3 & 2.1038 & 7.7432 & 2.1799 \\
\hline Reference & & & \\
Sladek et al. [23] & 2.105 & - & - \\
\hline
\end{tabular}

\subsection{Edged crack in an open-end wrench}

To illustrate the advantageous feature of the proposed method for solving practical problems, we consider the problem of an open-end wrench with a crack emanated from the side of the wrench handle as shown schematically in Fig. 7. The crack has a length of $a=w / 4$ where $w$ is the width of the handle. The working condition of the wrench is approximately modeled by applying a shear traction on the end of the handle

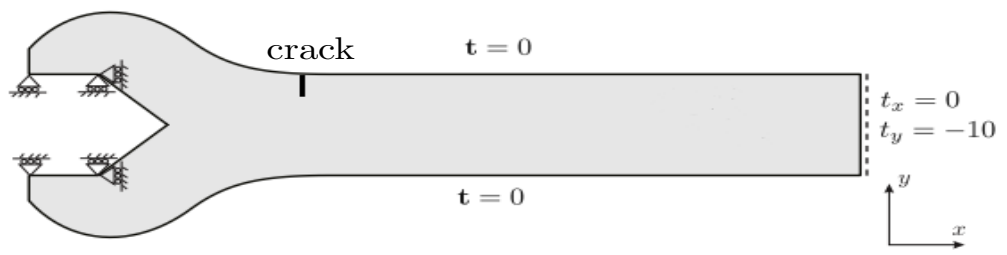

Fig. 7. Open-end wrench with an edged crack

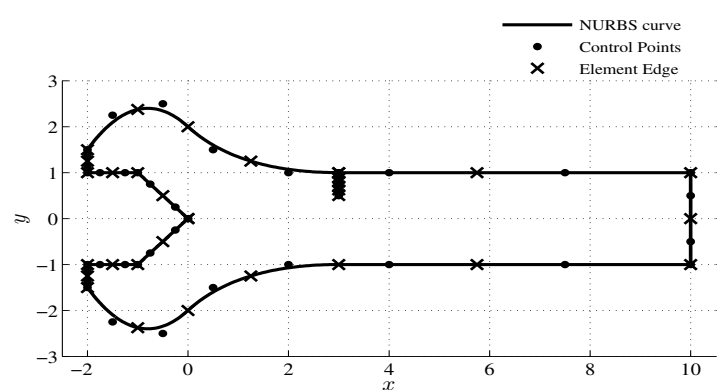

(a) Mesh 1: 37 control points for the boundary and 9 control points for the crack

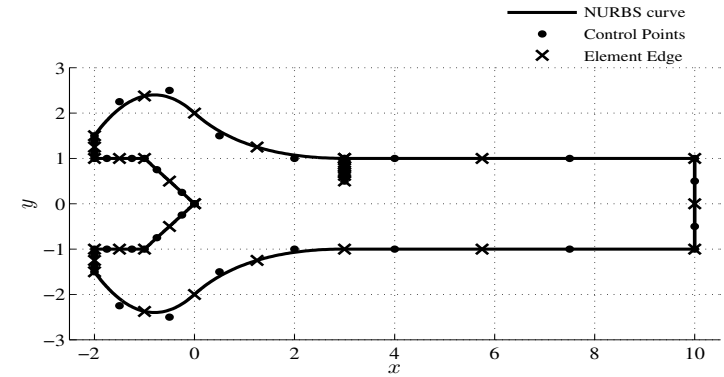

(b) Mesh 2: 37 control points for the boundary and 11 control points for the crack

Fig. 8. Meshes employed for the problem shown in Fig. 7 
and setting constraints on the jaws of the wrench as shown in the figure. Same as previous examples, the material employed for this example is $\mathrm{BaTiO}_{3}-\mathrm{CoFe}_{2} \mathrm{O}_{4}$ [22] with the properties shown in Tab.1. Two meshes shown in Fig. 8 are employed for the analysis. For both meshes, 37 control points are used to describe the boundary. To describe the crack, we use 9 control points for mesh 1 and 11 control points for mesh 2 . The results of normalized stress, electric displacement and magnetic induction intensity factors are presented in Tab. 3 in which $t_{y}$ is the value of applied shear traction, $a$ is the crack length, $D^{*}$ and $B^{*}$ are the same as in the previous example.

Table 3. Normalized stress, electric displacement and magnetic induction intensity factors for the problem shown in Fig. 7

\begin{tabular}{ccccc}
\hline Mesh \# & $\frac{K_{I}}{\left|t_{y}\right| \sqrt{\pi a}}$ & $\frac{K_{I I}}{\left|t_{y}\right| \sqrt{\pi a}}$ & $\begin{array}{c}\frac{K_{I V}}{D^{*} \sqrt{\pi a}} \\
\left(\times 10^{-10}\right)\end{array}$ & $\begin{array}{c}\frac{K_{V}}{B^{*} \sqrt{\pi a}} \\
\left(\times 10^{-9}\right)\end{array}$ \\
\hline 1 & 22.0384 & 1.0714 & -0.2245 & 0.5730 \\
2 & 22.0352 & 1.0710 & -0.2226 & 0.5742 \\
\hline
\end{tabular}

\section{CONCLUSION}

The IGA-SGBEM has been developed for the analysis of crack problems in twodimensional magneto-electro-elastic media. In the method, NURBS basis functions are employed to approximate both the geometry and the field variables of the integral equations that govern the problem. Because of that, the method does not need the geometric discretization but reather use directly the data transferred from a CAD model. Examples of crack in infinite and finite domains are examined and excellent agreement between the results computed by our developed method and other references are observed.

A significant application of the proposed method is to model crack propagation in magneto-electro-elastic media. This application could be implemented in 2D context without any considerable effort since the growth of 2D cracks can be easily modeled by either adding new elements in front of crack tips or stretching crack-tip element. However, a proper criterion for crack growth initiation has not been clearly identified. This could be a direction for our future work.

\section{ACKNOWLEDGMENT}

This research is funded by Vietnam National Foundation for Science and Technology Development (NAFOSTED) under grant number 107.02-2014.16.

\section{REFERENCES}

[1] T. J. R. Hughes, J. A. Cottrell, and Y. Bazilevs. Isogeometric analysis: CAD, finite elements, NURBS, exact geometry and mesh refinement. Computer Methods in Applied Mechanics and Engineering, 194, (39), (2005), pp. 4135-4195. doi:10.1016/j.cma.2004.10.008. 
[2] J. A. Cottrell, T. J. R. Hughes, and Y. Bazilevs. Isogeometric analysis: toward integration of CAD and FEA. John Wiley \& Sons, (2009).

[3] A. Rosolen and M. Arroyo. Blending isogeometric analysis and local maximum entropy meshfree approximants. Computer Methods in Applied Mechanics and Engineering, 264, (2013), pp. 95-107. doi:10.1016/j.cma.2013.05.015.

[4] N. Valizadeh, Y. Bazilevs, J. S. Chen, and T. Rabczuk. A coupled IGA-Meshfree discretization of arbitrary order of accuracy and without global geometry parameterization. Computer Methods in Applied Mechanics and Engineering, 293, (2015), pp. 20-37. doi:10.1016/j.cma.2015.04.002.

[5] S. S. Ghorashi, N. Valizadeh, and S. Mohammadi. Extended isogeometric analysis for simulation of stationary and propagating cracks. International Journal for Numerical Methods in Engineering, 89, (9), (2012), pp. 1069-1101. doi:10.1002/nme.3277.

[6] S. S. Ghorashi, N. Valizadeh, S. Mohammadi, and T. Rabczuk. T-spline based XIGA for fracture analysis of orthotropic media. Computers \& Structures, 147, (2015), pp. 138-146. doi:10.1016/j.compstruc.2014.09.017.

[7] T. Q. Bui. Extended isogeometric dynamic and static fracture analysis for cracks in piezoelectric materials using NURBS. Computer Methods in Applied Mechanics and Engineering, 295, (2015), pp. 470-509. doi:10.1016/j.cma.2015.07.005.

[8] R. N. Simpson, S. P. A. Bordas, J. Trevelyan, and T. Rabczuk. A two-dimensional isogeometric boundary element method for elastostatic analysis. Computer Methods in Applied Mechanics and Engineering, 209, (2012), pp. 87-100. doi:10.1016/j.cma.2011.08.008.

[9] B. Marussig, G. Beer, and C. Duenser. Isogeometric boundary element method for the simulation in tunneling. Applied Mechanics \& Materials, (553), (2014). doi:10.4028/www.scientific.net/amm.553.495.

[10] B. Marussig, J. Zechner, G. Beer, and T.-P. Fries. Fast isogeometric boundary element method based on independent field approximation. Computer Methods in Applied Mechanics and Engineering, 284, (2015), pp. 458-488. doi:10.1016/j.cma.2014.09.035.

[11] M. A. Scott, R. N. Simpson, J. A. Evans, S. Lipton, S. P. A. Bordas, T. J. R. Hughes, and T. W. Sederberg. Isogeometric boundary element analysis using unstructured Tsplines. Computer Methods in Applied Mechanics and Engineering, 254, (2013), pp. 197-221. doi:10.1016/j.cma.2012.11.001.

[12] R. N. Simpson, M. A. Scott, M. Taus, D. C. Thomas, and H. Lian. Acoustic isogeometric boundary element analysis. Computer Methods in Applied Mechanics and Engineering, 269, (2014), pp. 265-290. doi:10.1016/j.cma.2013.10.026.

[13] Y. J. Wang and D. J. Benson. Multi-patch nonsingular isogeometric boundary element analysis in 3D. Computer Methods in Applied Mechanics and Engineering, 293, (2015), pp. 71-91. doi:10.1016/j.cma.2015.03.016.

[14] Y. Wang, D. J. Benson, and A. P. Nagy. A multi-patch nonsingular isogeometric boundary element method using trimmed elements. Computational Mechanics, 56, (1), (2015), pp. 173191. doi:10.1007/s00466-015-1165-y.

[15] A. Frangi and G. Novati. Symmetric BE method in two-dimensional elasticity: evaluation of double integrals for curved elements. Computational Mechanics, 19, (2), (1996), pp. 58-68. doi:10.1007/bf02757784.

[16] A. Frangi and G. Novati. Regularized symmetric Galerkin BIE formulations in the Laplace transform domain for 2D problems. Computational Mechanics, 22, (1), (1998), pp. 50-60. doi:10.1007/s004660050338. 
[17] H. D. Tran and M. E. Mear. A weakly singular SGBEM for analysis of two-dimensional crack problems in multi-field media. Engineering Analysis with Boundary Elements, 41, (2014), pp. 60-73. doi:10.1016/j.enganabound.2014.01.003.

[18] C.-F. Gao, H. Kessler, and H. Balke. Crack problems in magnetoelectroelastic solids. Part I: exact solution of a crack. International Journal of Engineering Science, 41, (9), (2003), pp. 969 981. doi:10.1016/s0020-7225(02)00323-3.

[19] C.-F. Gao, H. Kessler, and H. Balke. Crack problems in magnetoelectroelastic solids. Part II: general solution of collinear cracks. International Journal of Engineering Science, 41, (9), (2003), pp. 983-994. doi:10.1016/s0020-7225(02)00324-5.

[20] B.-L. Wang and Y.-W. Mai. Fracture of piezoelectromagnetic materials. Mechanics Research Communications, 31, (1), (2004), pp. 65-73. doi:10.1016/j.mechrescom.2003.08.002.

[21] W.-Y. Tian and U. Gabbert. Multiple crack interaction problem in magnetoelectroelastic solids. European Journal of Mechanics-A/Solids, 23, (4), (2004), pp. 599-614. doi:10.1016/j.euromechsol.2004.02.002.

[22] F. Garcia-Sanchez, R. Rojas-Diaz, A. Sáez, and C. Zhang. Fracture of magnetoelectroelastic composite materials using boundary element method (BEM). Theoretical and Applied Fracture Mechanics, 47, (3), (2007), pp. 192-204. doi:10.1016/j.tafmec.2007.01.008.

[23] J. Sladek, V. Sladek, P. Solek, and E. Pan. Fracture analysis of cracks in magnetoelectro-elastic solids by the MLPG. Computational Mechanics, 42, (5), (2008), pp. 697-714. doi:10.1007/s00466-008-0269-z.

[24] T. Q. Bui and C. Zhang. Analysis of generalized dynamic intensity factors of cracked magnetoelectroelastic solids by X-FEM. Finite Elements in Analysis and Design, 69, (2013), pp. 19-36. doi:10.1016/j.finel.2013.02.001.

[25] T. Q. Bui, S. Hirose, C. Zhang, T. Rabczuk, C.-T. Wu, T. Saitoh, and J. Lei. Extended isogeometric analysis for dynamic fracture in multiphase piezoelectric/piezomagnetic composites. Mechanics of Materials, 97, (2016), pp. 135-163. doi:10.1016/j.mechmat.2016.03.001.

[26] J. Lei, C. Zhang, and T. Q. Bui. Transient dynamic interface crack analysis in magnetoelectroelastic bi-materials by a time-domain BEM. European Journal of Mechanics-A/Solids, 49, (2015), pp. 146-157. doi:10.1016/j.euromechsol.2014.07.010.

[27] B. H. Nguyen, H. D. Tran, C. Anitescu, X. Zhuang, and T. Rabczuk. An isogeometric symmetric Galerkin boundary element method for two-dimensional crack problems. Computer Methods in Applied Mechanics and Engineering, 306, (2016), pp. 252-275. doi:10.1016/j.cma.2016.04.002.

[28] H. D. Tran and M. E. Mear. Regularized boundary integral equations for two-dimensional crack problems in multi-field media. International Journal of Fracture, 181, (1), (2013), pp. 99 113. doi:10.1007/s10704-013-9823-6.

[29] H. D. Tran. A computational procedure for analysis of fractures in two-dimensional multi-field media. PhD thesis, The University of Texas at Austin, (2010). 